\title{
Primary percutaneous coronary intervention for patients with acute ST elevation myocardial infarction with and without diabetes mellitus
}

\author{
R J van der Schaaf, J P S Henriques, J J Wiersma, K T Koch, J Baan Jr, K J J Mulder, J D Durrer, \\ J G P Tijssen, J J Piek, R J de Winter
}

$\mathrm{P}$ atients with acute ST segment elevation myocardial infarction (STEMI) with diabetes mellitus (DM) have an increased mortality and morbidity when compared with patients without DM. ${ }^{1}$ Information is limited about clinical outcome of with STEMI and DM treated with reperfusion. Furthermore, patients with DM have a higher rate of thrombolysis failure. ${ }^{2}{ }^{3}$ Information is also limited on long term outcome of patients with DM treated with primary percutaneous coronary intervention (PCI) ${ }^{4}$ which is the reperfusion treatment of choice. ${ }^{5}$

We therefore studied one year mortality among patients with and without DM in a large cohort of patients with acute STEMI treated with primary PCI. Furthermore, we studied the impact of preadmission treatment for DM, either oral medication or with insulin.

\section{METHODS}

Between January 1997 and December 2002, 1463 consecutive and unselected patients were admitted to our hospital with acute STEMI. Of the 1463 patients, 17 were lost to follow up and for another 138 patients data about DM status was not available. The remaining 1308 patients constituted the study cohort. Follow up information was obtained one year after the initial event by written questionnaire sent to all patients. If necessary outpatients' reports were reviewed and general practitioners were contacted by telephone. Baseline clinical and angiographic data were collected prospectively on a dedicated database. The patients were categorised as being without DM or as having an established diagnosis of DM at admission. Patients with DM were categorised according to preadmission treatment: either with oral medication or diet controlled DM (non-insulin dependent DM (NIDDM)) or with insulin (insulin dependent DM (IDDM)). IDDM comprised both type 1 and type 2 DM. Patients treated with insulin in combination with oral medication were categorised as having IDDM.

To find independent predictors of one year mortality we performed multivariate analysis with a Cox proportional hazards regression model.

\section{RESULTS}

Of the 1308 patients, 174 (13.3\%) had a confirmed diagnosis of DM at admission. Patients with DM were older $(<60$ years; $44.4 \% \vee 64.4 \%, \mathrm{p}<0.001)$ and fewer of them were men $(64.9 \% v 74.9 \%, p=0.006)$ or smokers $(34.5 \% v 55.2 \%$, $\mathrm{p}<0.001)$. More of the patients with DM were hypertensive $(49.4 \% \vee 31.7 \%, \mathrm{p}<0.001)$, had a previous coronary event (previous PCI, STEMI, or coronary bypass grafting: $40.8 \% v$ $20.3 \%, \mathrm{p}<0.001$ ), or had a long ischaemic time ( $>3$ hours) $(54.7 \% \vee 44.3 \%, p=0.027)$ compared with patients without DM. Patients with DM had a higher incidence of cardiogenic shock at admission $(13.2 \% \vee 8.3 \%, p=0.034)$ and more of them needed intra-aortic balloon counter pulsation ( $12.1 \% v$ $5.6 \%, \mathrm{p}<0.001)$. Patients with DM had more multivessel disease $(55.7 \% \vee 37.9 \%, \mathrm{p}<0.001)$ and coronary calcifications $(27.6 \% \vee 19.5 \%, \mathrm{p}=0.014)$ but fewer successful PCI procedures $(86.6 \% \quad v \quad 95.2 \%, \mathrm{p}<0.001)$ compared with patients without DM. Mortality after one year's follow up was $8.9 \%(n=113)$ in the whole cohort, $17.8 \%$ for patients with DM, and $7.2 \%$ for patients without DM (OR 2.9, 95\% CI 1.8 to $4.3, \mathrm{p}<0.001)$. Of the 174 patients with DM, 126 $(72.4 \%)$ were taking oral treatment (NIDDM) and $48(27.8 \%)$ were taking insulin (IDDM) at admission. Baseline and angiographic characteristics did not differ between patients with IDDM and those with NIDDM. IDDM patients tended to have more coronary calcifications (37.5\% v 23.8\%, $\mathrm{p}=0.071)$ and less successful PCI $(81.3 \% \quad v \quad 88.9 \%$, $\mathrm{p}=0.184$ ) than patients with NIDDM. Mortality was $27.1 \%$ for patients with IDDM and $14.3 \%$ for patients with NIDDM (OR 2.2, 95\% CI 1.0 to 5.0, p < 0.05). Multivariate analysis showed that significant independent predictors for mortality were cardiogenic shock at presentation, PCI failure, IDDM, NIDDM, and coronary calcifications (table 1).

\section{DISCUSSION}

This study confirms that even with the reperfusion treatment of choice, patients with STEMI and DM have a greater long term mortality than do patients without DM. They have different baseline characteristics from patients without DM. Patients with DM also present with a higher incidence of cardiogenic shock, possibly at least partly due to a longer ischaemic time. Furthermore, more patients with than without DM had PCI failure possibly also as a consequence of a longer ischaemic time. Some angiographic differences may also partly explain the difference in PCI success. Both coronary calcifications and a higher rate of multivessel disease are also associated with more complicated PCI procedures and were more common among patients with DM.

The second finding is that preadmission treatment for DM is an independent predictor for one year mortality. Patients requiring insulin have longstanding type $1 \mathrm{DM}$ or type $2 \mathrm{DM}$ without adequate glycaemic regulation with oral medication. These patients with IDDM mostly have longstanding DM and mortality of those with type 2 DM receiving insulin at admission is about four times higher than that of patients without DM and two times higher than that of patients with DM taking oral medication at admission. In our study about $13 \%$ of the patients had a previous diagnosis of DM. Of all the

Abbreviations: DM, diabetes mellitus; IDDM, insulin dependent diabetes mellitus; NIDDM, non-insulin dependent diabetes mellitus; PCI, percutaneous coronary intervention; STEMI, ST segment elevation myocardial infarction 
Table 1 Independent predictors of one year mortality in 1308 patients with ST elevation myocardial infarction treated with primary percutaneous coronary intervention

\begin{tabular}{|c|c|c|c|}
\hline & $\mathbf{R R}^{*}$ & $95 \% \mathrm{Cl}$ & p Value \\
\hline Shock at presentation $†$ & 5.405 & 3.311 to 8.771 & $<0.001$ \\
\hline $\mathrm{PCl}$ failure† & 3.127 & 1.586 to 6.166 & 0.001 \\
\hline IDDM†‡ & 3.246 & 1.219 to 8.695 & 0.018 \\
\hline NIDDM†‡ & 2.673 & 1.248 to 5.714 & 0.011 \\
\hline Coronary calcification $†$ & 1.680 & 1.016 to 2.777 & 0.043 \\
\hline Family history & 1.452 & 0.880 to 2.396 & 0.144 \\
\hline Multivessel disease & 1.412 & 0.869 to 2.293 & 0.163 \\
\hline Age $>60$ years & 1.402 & 0.808 to 2.439 & 0.229 \\
\hline Men & 1.206 & 0.717 to 2.028 & 0.479 \\
\hline Ischaemic time $>180 \mathrm{~min}$ & 1.206 & 0.766 to 1.901 & 0.417 \\
\hline Previous event§ & 1.184 & 0.710 to 1.976 & 0.515 \\
\hline Hypertension & 1.082 & 0.662 to 1.766 & 0.753 \\
\hline Smoking & 0.801 & 0.493 to 1.302 & 0.371 \\
\hline Hypercholesterolaemia & 0.704 & 0.393 to 1.261 & 0.238 \\
\hline
\end{tabular}

${ }^{*}$ Cox regression analysis; $†$ significant predictor; $\ddagger$ diabetes was entered as a categorical variable (no diabetes, NIDDM, or IDDM); §previous PCI, STEMI, or coronary artery bypass graffing.

$\mathrm{Cl}$, confidence interval; IDDM, insulin dependent diabetes mellitus (patients treated with insulin); NIDDM, non-insulin dependent diabetes mellitus (patients treated orally); RR, relative risk.

patients with DM about $28 \%$ were taking insulin at admission, in agreement with large registries.

As a limitation, we did not routinely estimate haemoglobin $\mathrm{A}_{\mathrm{IC}}$ or test for DM on admission or during follow up. Despite the relatively large sample size, the absolute number of patients in each DM category is somewhat small. Only 23\% of all patients were treated with abciximab.

Patients with acute STEMI with diagnosed DM more often present with cardiogenic shock and have more severe coronary disease and PCI failure. After primary PCI for STEMI, the one year mortality rate is $7.2 \%$ for patients without DM and $17.8 \%$ for patients with DM. For patients with DM taking oral medication at the time of admission for
STEMI, the one year mortality is $14.3 \%$ and for those taking insulin mortality is $27.1 \%$. Preadmission treatment for DM is an independent predictor of one year mortality. Even after primary PCI for acute STEMI, patients with DM treated with insulin form a subgroup with a mortality about four times higher than that of patients without DM and two times higher than that of patients with DM treated with oral medication in daily, real life clinical practice.

\section{Authors' affiliations}

R J van der Schaaf, J P S Henriques, J J Wiersma, K T Koch, J Baan Jr, K J J Mulder, J D Durrer, J G P Tijssen, J J Piek, R J de Winter, Department of Cardiology, Academic Medical Centre, Amsterdam, the Netherlands

There are no conflicts of interest or financial disclosure

Correspondence to: Dr José P S Henriques, Department of Cardiology, Academic Medical Centre, Meibergdreef 9, 1105 AZ Amsterdam, Netherlands; i.p.henriques@amc.uva.nl

Accepted 4 May 2005

\section{REFERENCES}

1 Mak KH, Moliterno DJ, Granger CB, et al. Influence of diabetes mellitus on clinical outcome in the thrombolytic era of acute myocardial infarction. GUSTO-I Investigators. Global utilization of streptokinase and tissue plasminogen activator for occluded coronary arteries. J Am Coll Cardiol 1997; 30:171-9.

2 Hsu LF, Mak KH, Lau KW, et al. Clinical outcomes of patients with diabetes mellitus and acute myocardial infarction treated with primary angioplasty or fibrinolysis. Heart 2002;88:260-5.

3 Zairis MN, Lyras AG, Makrygiannis SS, et al. Type 2 diabetes and intravenous thrombolysis outcome in the setting of ST elevation myocardial infarction. Diabetes Care 2004;27:967-71.

4 Timmer JR, Ottervanger JP, Thomas K, on behalf of the Zwolle myocardial infarction study group, et al. Long-term, cause-specific mortality after myocardial infarction in diabetes. Eur Heart J 2004;25:926-31.

5 Keeley EC, Boura JA, Grines CL. Primary angioplasty versus intravenous thrombolytic therapy for acute myocardial infarction: a quantitative review of 23 randomised trials. Lancet 2003;361:13-20.

\section{IMAGES IN CARDIOLOGY}

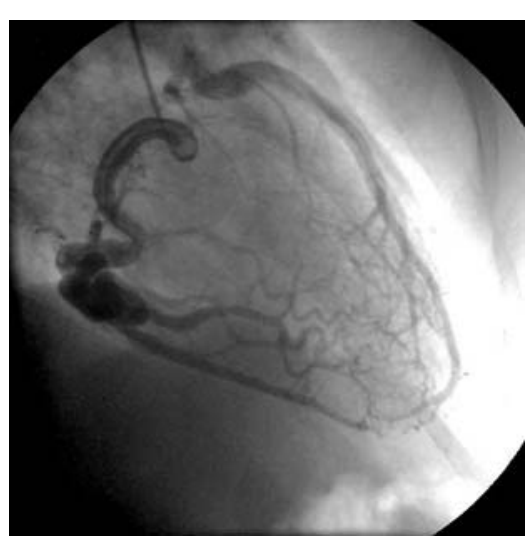

52 year old man presented with near syncope while standing in a bus. An ECG showed widespread T wave inversion involving lead Vl-4, I, aVL. Myocardial infarction was ruled out on subsequent cardiac enzyme assays. A dobutamine stress echocardiogram showed abnormal wall motion in the left anterior descending artery territory during stress. Cardiac catheterisation showed a large right coronary artery collateralising the left coronary artery, which emptied into the pulmonary artery (panel). There was no significant coronary artery disease and the left ventricular systolic function was normal. The calculated left to right shunt (Qp:Qs ratio) was 1.07 .

Anomalous left coronary artery from the pulmonary artery is a rare, congenital cardiac anomaly accounting for approximately $0.25-0.5 \%$ of all congenital heart disease. It is usually an isolated cardiac anomaly but, in rare incidences, has been described with patent ductus arteriosus, ventricular septal defect, tetralogy of Fallot, and coarctation of the aorta. Collateral circulation between the right and left coronary systems ensues after birth and the left ventricular myocardium is chronically underperfused, since flow is preferentially directed into the pulmonary vascular bed and away from the left ventricular myocardium due to the low pulmonary vascular resistance (coronary steal phenomena). Left untreated, the mortality rate in the first year of life is $90 \%$ secondary to myocardial ischaemia or infarction and congestive heart failure. In rare cases, the clinical presentation of myocardial ischaemia may be delayed into early childhood, or even adulthood as in our patient, when intracardiac shunt is minimal. 